\title{
Análise da integração ensino-serviço para a formação de residentes em medicina de família e comunidade
}

\section{Analysis of the teaching-service integration for training residents in family and community medicine}

\author{
Anderson Milfont Feitosa de Oliveira' 10 | andersonmilfont@gmail.com \\ Maria Rosilene Cândido Moreira² (1) rosilene.moreira@ufca.edu.br \\ Samyra Paula Lustoza Xavier ${ }^{3}$ (1) samyralustoza@gmail.com \\ Maria de Fátima Antero Sousa Machado' 10 fatimaantero@uol.com.br
}

\begin{abstract}
RESUMO
Introdução: A integração entre as instituições de ensino e os serviços de saúde na formação de residentes médicos requer mudanças em ambas as partes envolvidas, principalmente no cenário assistencial, fazendo-se necessária remodelação da cultura dos atores sociais que constituem o SUS.
\end{abstract}

Objetivo: O objetivo deste estudo foi efetuar, pelo mapeamento das forças, fraquezas, oportunidades e ameaças, o diagnóstico situacional da integração ensino-serviço em um Programa de Residência em Medicina de Família e Comunidade (PRMFC).

Método: Trata-se de pesquisa-intervenção com preceptores e gestores de saúde de um município do estado do Ceará, no Brasil. Os dados foram coletados pela entrevista semiestruturada, submetidos à Classificação Hierárquica Descendente (CHD) com auxílio do software IRaMuTeQ, analisados conforme eixos da matriz SWOT (forças, fraquezas, oportunidades, ameaças) e discutidos com apoio da literatura.

Resultados: As classes de cada CHD originaram os fatores de cada eixo da matriz SWOT, delineando como pontos fortes: parceria entre os agentes de integração e inclusão do residente no cotidiano das equipes; pontos fracos: distanciamento entre preceptor e instituição de ensino e ausência de estratégias de valorização da preceptoria; oportunidades: perspectiva de o programa servir como ferramenta de gestão da saúde; e ameaças: problemas na gestão dos equipamentos da saúde, com reflexos sobre a estrutura dos serviços, gestão do programa e qualidade da formação.

Conclusões: A análise da matriz SWOT viabilizou a identificação de pontos fortes e fragilidades no PRMFC, viabilizando a elaboração de estratégias para o fortalecimento da integração ensino-serviço, na perspectiva de melhoria da gestão, formação e execução do cuidado em saúde.

Palavras-chave: Integração Docente-Assistencial; Capacitação de Recursos Humanos em Saúde; Residência Médica; Medicina de Família e Comunidade; Saúde Coletiva.

\begin{abstract}
Introduction: Integration between educational institutions and health services in the training of medical residents requires changes on both sides, especially in the care setting, making it necessary to remodel the culture of the social actors that make up the SUS.

Objective: The objective of this study was to performa situational diagnosis of the teaching-service integration in a Residency Program in Family and Community Medicine (PRMFC) by mapping the reported strengths, weaknesses, opportunities and threats.

Method: Intervention research with preceptors and health managers in a municipality in the state of Ceará, Brazil. Data were collected through semistructured interviews, submitted to the Descending Hierarchical Classification (DHC) with the aid of the IRaMuTeQ software, analyzed according to the axes of the SWOT matrix (strengths, weaknesses, opportunities, threats) and discussed with the support of literature.

Results: Each DHC class generated the factors of each axis of the SWOT matrix, outlining as strong points: partnership between the agents of integration and inclusion of the resident in the daily routines of the teams; weaknesses: distance between preceptor and educational institution and absence of strategies for valuing preceptorship; opportunities: perspective of the Program to serve as a health management tool; and threats: problems in the management of health equipment, reflecting on the structure of services, Program management and quality of training.
\end{abstract}

Conclusions: The analysis of the SWOT matrix enabled the identification of strengths and weaknesses in the PRMFC, supporting the development of strategies to strengthen teaching-service integration, with a view to improving health care management, training and execution.

Keywords: Teaching Care Integration; Training of Human Resources in Health; Medical Residency; Family and Community Medicine; Public Health.

${ }^{1}$ Fundação Oswaldo Cruz, Eusébio, Ceará, Brasil.

2 Universidade Federal do Cariri, Barbalha, Ceará, Brasil.

${ }^{3}$ Universidade Regional do Cariri, Iguatu, Ceará, Brasil.

Editora-chefe: Daniela Chiesa

Editor associado: Roberto Zonato Esteves

Recebido em 11/08/20; Aceito em 04/11/20.

Avaliado pelo processo de double blind review. 


\section{INTRODUÇÃO}

Desde a implantação do Sistema Único de Saúde (SUS), a formação profissional tem sido discutida como um aspecto central para o alcance e a efetivação das políticas públicas em saúde, uma vez que o modelo tradicional de ensino biologicista, curativista e hospitalocêntrico - já não atende às atuais demandas sociais.

Mudanças efetivas na formação profissional requerem a transformação de práticas antidemocráticas e não integradoras, para que haja a incorporação de princípios de integralidade no sentido amplo da dimensão pessoal/profissional, da organização dos serviços e do desenvolvimento de políticas centradas na necessidade dos usuários ${ }^{1}$.

Nessa perspectiva, viu-se a imperativa necessidade de ampliar a cobertura de serviços de saúde, observada pela escassez de profissionais médicos graduados e especialistas no SUS. Assim, como forma de superar tal problema, o governo federal institui o Projeto Mais Médicos para o Brasil (PMMB), que se apresenta como importante política indutora para o aumento no número de vagas de cursos de graduação e de programas de residência médica (RM) em localidades e especialidades de necessidade nacional ${ }^{2,3}$.

Os desafios são inúmeros com tamanha dimensão da proposta trazida pelo PMMB, pois projeta para o futuro o provimento de médicos para o SUS com problemas não resolvidos no passado tanto na educação quanto na gestão e na atenção à saúde, e principalmente na política de formação profissional, como o trabalho em equipe multiprofissional e a formação docente do trabalhador do SUS ${ }^{4}$. As potencialidades abertas pela implantação dessa nova política, com alto grau de complexidade de gestão nas diversas instâncias, além das potenciais insuficiências dos serviços assistenciais, evidenciam um novo momento nos processos de integração ensino-serviço ${ }^{5,6}$.

Essa integração entre as instituições de ensino superior (IES) e os serviços de saúde requer mudanças em ambas as partes envolvidas, principalmente no cenário assistencial, fazendo-se necessária uma remodelação da cultura dos atores sociais que constituem o SUS, com ênfase nos seus gestores, a fim de compreendê-lo como escola, ou seja, estruturar suas ações dentro do eixo da integralidade, de modo a buscar efetivamente a integração entre ensino e serviço'. Nesse contexto, cada vez mais se observa a necessidade do papel do Estado em ordenar seus processos de formação na saúde a fim de que regule e intermedeie a integração ensino-serviço entre os serviços de saúde nas diversas esferas governamentais (municipal, estadual e federal) e as entidades formadoras.

Com base nessas considerações, o objetivo deste estudo consistiu em efetuar, pelo mapeamento das forças, fraquezas, oportunidades e ameaças, o diagnóstico situacional da integração ensino-serviço em um Programa de Residência em Medicina de Família e Comunidade (PRMFC).

\section{MÉTODO}

Trata-se de pesquisa-intervenção com desenho participativo, pois estudos desse tipo visam unir teoria e prática, em que a construção de conhecimentos sobre a realidade local e o estímulo de uma postura proativa voltada para o desenvolvimento configuram-se na perspectiva de resultar em instrumento científico e pedagógico para a transformação local. Nesse contexto, estabelece-se uma relação de confiança mútua $^{7}$, na qual pesquisador e pesquisado relacionam-se de modo colaborativo, ou seja, ambos adquirem resultado a ser apropriado por meio do estudo realizado ${ }^{8}$.

A pesquisa foi realizada no município de Juazeiro do Norte, com quatro integrantes da gestão municipal da saúde e dois preceptores do PRMFC pertencentes ao serviço de saúde e à instituição de saúde concomitantemente, escolhidos, de forma intencional, por serem os atores (informantes-chave) que estão diretamente relacionados à relação ensino-serviço-gestão.

Os dados foram coletados entre setembro e novembro de 2018 mediante entrevista semiestruturada, cujas perguntas contemplaram aspectos referentes à percepção dos preceptores sobre o campo de práticas e o processo de integração ensino-serviço, enquanto aos gestores couberam questões concernentes à relação da gestão municipal com a coordenação do PRMFC.

O material obtido foi lido e organizado por conteúdos semânticos conforme os eixos da matriz SWOT - acrônimo de strengths (forças), weaknesses (fraquezas), opportunities (oportunidades) e threats (ameaças) -, originando quatro corpus que formaram o banco de dados (corpora) para análise deste estudo.

A análise dos corpora ocorreu com auxílio do software Interface de $R$ pour $L$ Analyses Multidimensionnelles de Textes $\mathrm{L}$ de Questionnaires (IRaMuTeQ) versão 0.7 alfa 2. Trata-se de um programa gratuito, do tipo open source, desenvolvido na França em 2009 e utilizado em pesquisas no Brasil a partir de 2013, contendo dicionário de palavras em idioma português 9 . Utiliza linguagem python e realiza cálculos estatísticos pela interconexão com a estrutura do software $\mathrm{R}$, caracterizando o rigor estatístico da pesquisa ${ }^{10}$ e viabilizando assim as diversas análises, das mais simples, como a lexicografia básica, até as mais complexas, como a Classificação Hierárquica Descendente (CHD) e a Nuvem de Palavras (NP) utilizadas neste estudo.

A CHD visa obter classes de segmentos de texto (ST) que, ao mesmo tempo, apresentam vocabulário semelhante entre si e diferente dos ST das outras classes, realizando análises quantitativas desses textos inseridos em seus 
múltiplos contextos e por classes de conteúdo ${ }^{11}$, pois considera que palavras usadas em contexto similar estão associadas ao mesmo mundo léxico, compondo mundos mentais específicos ou contextos semânticos de uma mesma representação. Destaca-se que, para esse tipo de análise ser considerado válido, é necessário o aproveitamento mínimo de 75\% dos ST (percentual de retenção). Caso não seja possível obter esse percentual, devem-se empregar outros tipos de análises que o programa oferece $^{12}$.

Já a NP viabiliza graficamente o agrupamento das palavras, considerando a frequência com que aparecem no material analisado9. Assim, quanto maior a palavra na nuvem, maior sua expressividade no corpus. Esse tipo de análise possibilita a rápida identificação visual das palavras centrais do material textual ${ }^{13}$, favorecendo uma compreensão global acerca do fenômeno estudado.

Após esse processo de classificação, o software organizou as análises em dendrogramas (um para cada corpus), que delinearam a divisão em classes, suas respectivas palavras e a frequência destas dentro da classe, assim como seu grau de significância (valor $p$ ) nessa classe, calculado pelo teste de qui-quadrado $\left(x^{2}\right)$, que estabelece a relação de associação estatística de cada palavra com a classe. Adicionalmente, para facilitar a rápida identificação dos termos centrais, uma NP foi gerada para cada classe da CHD.

As classes exibiram expressões que coadunaram significação e sentido, e, quando representadas nas NPs, possibilitaram a configuração dos fatores de cada eixo da matriz SWOT, favorecendo o conhecimento do panorama situacional dos ambientes interno e externo ao processo sob análise ${ }^{14}$.

Neste estudo, considerou-se ambiente interno (forças e fraquezas) os aspectos que envolveram a relação entre preceptores e residentes, e entre residentes e equipe de saúde. Ambiente externo (oportunidades e ameaças) foi caracterizado pelas questões envolvendo a gestão local de saúde, a gerência do PRMFC e contextos que influenciaram sua execução.

A matriz SWOT auxiliou a realização do diagnóstico situacional do PRMFC, fornecendo informações que possibilitaram a análise detalhada do cenário investigado e viabilizaram a elaboração de estratégias para o fortalecimento da integração ensino-serviço, que utilizou como referencial teórico-metodológico o Planejamento Estratégico Situacional ${ }^{15}$.

Para garantir o anonimato dos informantes, os gestores foram identificados pela letra $G$ e os preceptores pela letra $P$, além do número sequencial das entrevistas. O estudo obteve aprovação do Comitê de Ética em Pesquisa da Universidade Regional do Cariri, com número 2.005.435.

\section{RESULTADOS}

\section{Eixo forças}

O corpus forças foi dividido em 69 ST, e a CHD reteve 53 destes (76,81\% do total), originando seis classes. Inicialmente, dividiu-se o corpus em dois subcorpus (de um lado, as classes 1, 2 e 3, e, de outro, as demais); em seguida, a classe 1 foi separada das classes 2 e 3; no terceiro momento, a classe 6 foi separada das classes 4 e 5;no quarto momento, separaram-se as classes 2 e 3; e, na quinta partição, houve a separação das classes 4 e 5 , finalizando o processo.

A classe 4 (fator 1), com 20,8\% de representatividade do corpus, evidenciou como positiva a relação de "parceria" $\left(x^{2}=25,5 ; p<0,0001\right)$ entre os agentes de integração.

A classe 1 (fator 2), com 18,87\% de representação, apresentou a RMFC como o ambiente no qual ocorre a inclusão de "discussões" $\left(x^{2}=13,7 ; p=0,0002\right)$ acerca do perfil dos profissionais que se pretende formar, com base no contexto "diferente" $\left(x^{2}=10,1 ; p=0,001\right)$ de atuação, e conforme as necessidades do SUS.

Tanto a classe 6 como a classe 5 abrangeram, cada uma, $16,98 \%$ de representação do corpus. A classe 5 (fator 3) evidenciou ser um ponto forte quando os profissionais "acham" $\left(x^{2}=18,9 ; p<0,0001\right)$ importante a inserção do residente nas atividades cotidianas da "equipe" $\left(x^{2}=7,3 ; p=0,006\right)$, e a classe 6 (fator 4) destacou que essa inserção constitui fator relevante para o "residente" $\left(x^{2}=28,4 ; p<0,0001\right)$, em complementação à sua "formação" $\left(x^{2}=21,5 ; p<0,0001\right)$ médica e na atenção às "famílias" $\left(x^{2}=18,9 ; p<0,0001\right)$.

As classes 2 e 3 obtiveram o mesmo percentual de representatividade do corpus (13,21\%). A classe 2 (fator 5) destacou positivamente a realização de diálogo entre os profissionais do serviço e o residente, pois essas "conversas" $\left(x^{2}=20,9 ; p<0,0001\right)$ proporcionam ajustes no "ensino" $\left(x^{2}=23,5 ; p<0,0001\right)$ e no "serviço" $\left(x^{2}=19,9 ; p<0,0001\right)$, sendo corroborada pela classe 3 (fator 6), que evidenciou a presença do residente um estímulo para os profissionais de saúde, oferecendo oportunidades para "discutir" $\left(x^{2}=5,1 ; p=0,02\right)$ no "serviço" $\left(x^{2}=4,9 ; p=0,02\right)$ novas possibilidades na atenção à saúde.

As NPs elaboradas a partir das classes originadas e os relatos dos participantes ilustram os achados de maior expressividade deste corpus (Figura 1). 
Figura 1. Nuvens de Palavras das classes geradas na CHD do corpus forças

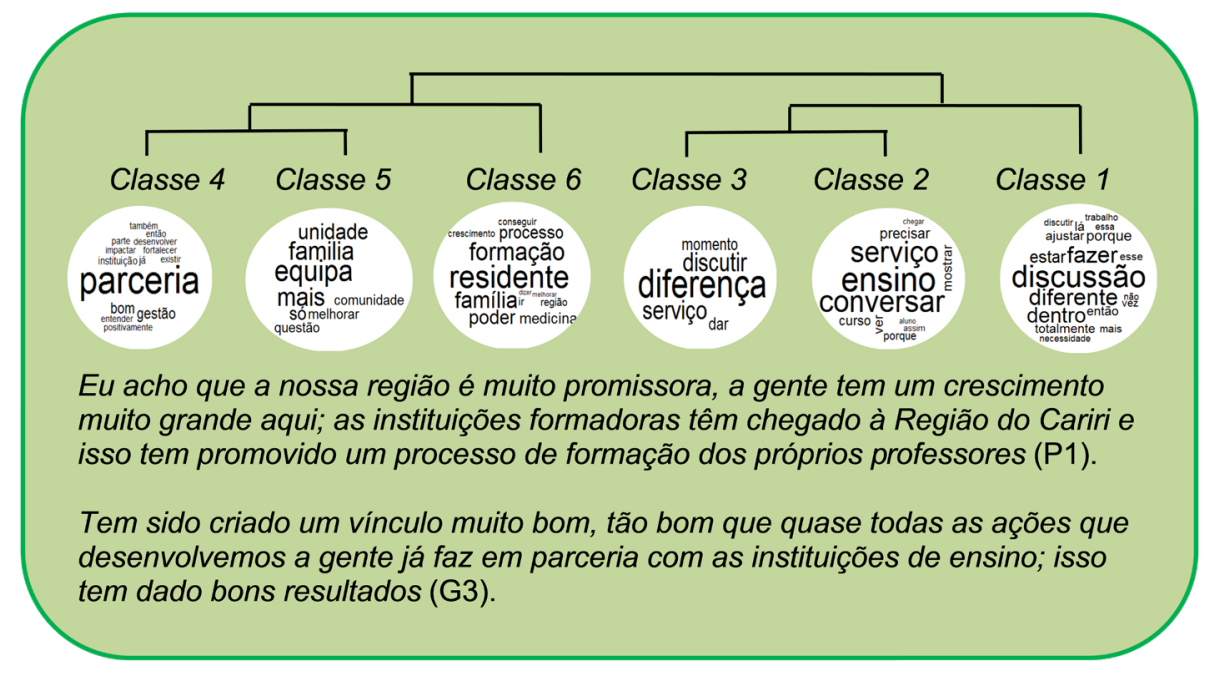

\section{Eixo fraquezas}

O corpus fraquezas foi dividido em $134 \mathrm{ST}$, com 82,09\% de retenção (110 ST), e resultou em sete classes. O corpus foi dividido inicialmente em dois subcorpus (de um lado, as classes 2 e 3, e, de outro,as demais); em seguida, as classes 1 e 6 foram separadas das demais; no terceiro momento, a classe 7 se diferenciou das classes 4 e 5; no quarto momento, separaramse as classes 2 e 3; na quinta partição, houve a separação das classes 1 e 6;e, na sexta partição, as classes 4 e 5 foram separadas, finalizando o processo.

A classe 4 (fator 1) obteve o maior percentual de representação do corpus (16,36\%), destacando como uma fraqueza o sentimento de distanciamento da instituição de ensino, por ela não estar "próximo" $\left(x^{2}=15,5 ; p<0,0001\right)$ dos médicos durante o exercício da preceptoria.

A classe 3 (fator 2) representou $15,45 \%$ do corpus, evidenciando a problemática da quantidade de "pacientes" $\left(x^{2}=47,5 ; p<0,0001\right)$ para "atender" $\left(x^{2}=41,2 ; p<0,0001\right)$, indicando que o dimensionamento da "demanda" $\left(x^{2}=25,1\right.$; $p<0,0001)$, para conferir mais efetividade às ações da preceptoria, necessita ser objeto de análise pelos agentes de integração.

As classes 1 e 7 obtiveram o mesmo percentual de representação do corpus (14,6\%). A classe 1 (fator 3 ) identificou como fraqueza a não utilização de indicadores para evidenciar a "real" ( $\left.x^{2}=18,1 ; p<0,0001\right)$ "necessidade" $\left(x^{2}=24,4 ; p<0,0001\right)$ do "serviço" ( $\left.x^{2}=27,2 ; p<0,0001\right)$ e assim balizar o planejamento de ações da residência médica; enquanto a classe 7 (fator 4) destacou como ponto fraco a ausência de iniciativas que a "gestão" ( $\left.x^{2}=18,3 ; p<0,0001\right)$ poderia "criar" $\left(x^{2}=30,5 ; p<0,0001\right)$ para "valorizar" ( $\left.x^{2}=30,8 ; p<0,0001\right)$ os preceptores.

As classes 5 e 6 também obtiveram o mesmo percentual de representação (13,64\%). A classe 5 (fator 5) fez emergir a problemática de os preceptores não estarem capacitados para a execução da "preceptoria" $\left(x^{2}=26,3 ; p<0,0001\right)$ e de esse "processo" $\left(x^{2}=9,4 ; p=0,002\right)$ "formador" $\left(x^{2}=12,0 ; p=0,0005\right)$ ser mantido sempre à "parte" $\left(x^{2}=34,3 ; p<0,0001\right)$ das discussões relacionadas ao PRMFC. Já a classe 6 (fator 6) apresentou como fraqueza que a compreensão dos profissionais acerca da integração ensino-serviço não tem sido "fácil" $\left(x^{2}=26,3\right.$; $p<0,0001)$, embora o PRMFC tenha passado a "existir" $\left(x^{2}=15,2\right.$; $\mathrm{p}<0,0001)$ na "região" $\left(x^{2}=13,3 ; p=0,0002\right)$ já há algum tempo.

A classe 2 (fator 7 ) representou $11,82 \%$ do corpus e evidenciou a difícil tarefa de incluir as estratégias de ensinoaprendizagem da preceptoria no conjunto das atividades cotidianas dos profissionais, resultando na ausência de algumas delas, tais como a inexistência de horário dedicado a estudos em grupo e discussão de casos, pois consideram que o volume de pessoas para "atendimento" $\left(x^{2}=28,3 ; p<0,0001\right)$ dificulta "fazer" $\left(x^{2}=28,3 ; p<0,0001\right)$ "discussão" $\left(x^{2}=28,3 ; p<0,0001\right)$ após cada "consulta" $\left(x^{2}=28,3 ; p<0,0001\right)$.

As NPs elaboradas a partir dessas classes e os discursos dos participantes clarificam os achados mais expressivos desse corpus (Figura 2).

\section{Eixo oportunidades}

O corpus oportunidades foi dividido em 122 ST e reteve 96 ST (78,69\% de retenção), originando seis classes. Na primeira partição do corpus, a classe 1 foi separada das demais; em seguida, as classes 2 e 3 foram separadas das demais; no terceiro momento, a classe 6 foi separada das classes 4 e 5; no quarto momento, separaram-se as classes 2 e 3; e, na quinta partição, houve a separação das classes 4 e 5 , finalizando o processo. 
Figura 2. Nuvens de Palavras das classes geradas na CHD do corpus fraquezas

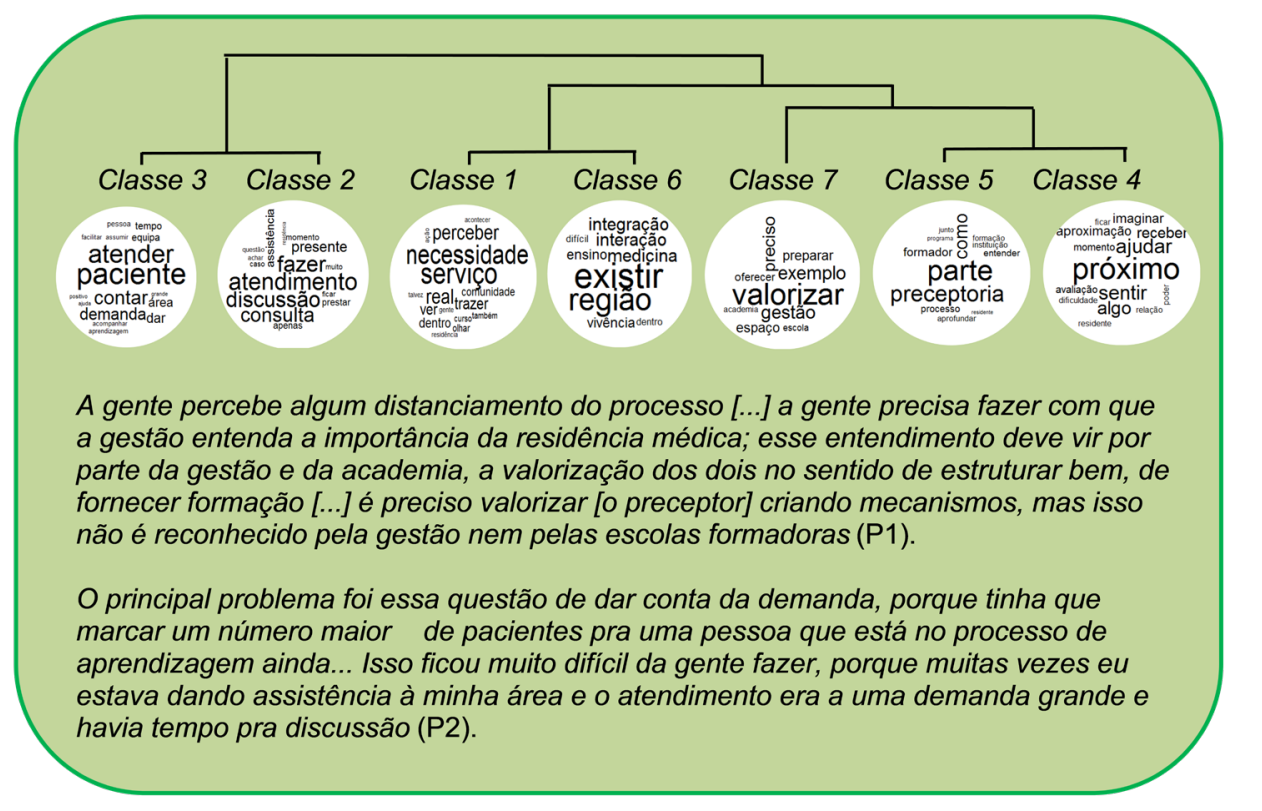

A classe 3 (fator 1) obteve 20,83\% de representação, destacando a percepção da necessidade da troca de experiências com outros preceptores como sendo potencial recurso para o fortalecimento do PRMFC, mediado pela responsabilidade de cuidar das "pessoas" $\left(x^{2}=17,5 ; p<0,0001\right)$ e assim "desenvolver" $\left(x^{2}=15,5 ; p<0,0001\right)$ um trabalho pautado no compromisso, no "querer" $\left(x^{2}=15,2 ; p<0,0001\right)$.

A classe 5 (fator 2) representou $18,75 \%$ do corpus, cujos relatos apontaram o PRMFC como ferramenta de gestão da saúde e benefício para a população, que ajuda as "famílias" $\left(x^{2}=13,4 ; p=0,0002\right)$ adscritas, com "ganho" $\left(x^{2}=12,9\right.$; $\mathrm{p}=0,0003$ ) para elas.

As classes 1 e 4 representaram 16,67\%, cada uma, do corpus. A classe 1 (fator 3 ) apresentou a necessidade de sensibilização dos gestores de saúde para o alinhamento entre ensino e serviço, partindo da análise dos "pontos" $\left(x^{2}=20,9\right.$; $p<0,0001)$ específicos de cada "lado" $\left(x^{2}=31,5 ; p<0,0001\right)$, em "conversas" $\left(x^{2}=20,9 ; \quad p<0,0001\right)$ mediadas pela "compreensão" $\left(x^{2}=20,5 ; p<0,0001\right)$ das "necessidades" $\left(x^{2}=15,5\right.$; $p<0,0001)$ de cada instituição envolvida.

A classe 4 (fator 4) evidenciou a realização de encontros de capacitação como estratégia de aprimoramento do PRMFC, tendo sido relatado por alguns participantes que isso já vem ocorrendo no município, com alcance positivo na reestruturação das "unidades" $\left(x^{2}=17,9 ; p<0,0001\right)$ e na "capacitação" $\left(x^{2}=15,5\right.$; $\mathrm{p}<0,0001)$ dos preceptores.

A classe 6 (fator 6), com 14,58\% de representação, destacou a formação permanente como uma estratégia para o fortalecimento do PRMFC, pois algumas experiências de capacitação realizadas no município foram bem-sucedidas, como a que ocorreu por meio da parceria com a "Escola de Saúde Pública" $\left(x^{2}=14,2 ; p<0,0001\right)$ do estado do Ceará. Corroborando esse resultado, a classe 2 (fator 5), com representação de 12,5\%, complementou a importância da formação permanente, por considerar que a "criação" $\left(x^{2}=16,8\right.$; $p<0,0001)$ de ambientes de "formação" $\left(x^{2}=20,1 ; p<0,0001\right)$ consiste em oportunidade para "avaliar" $\left(x^{2}=21,68 ; p<0,0001\right)$ os processos da residência médica.

As NPs e os relatos dos profissionais reforçaram os principais achados desse corpus (Figura 3).

\section{Eixo ameaças}

O corpus ameaças foi dividido em 41 ST, com percentual de retenção de $80,49 \%$ (33 ST), originando sete classes. A primeira partição do corpus originou a classe 2 em oposição às demais; em seguida, as classes 5 e 6 foram separadas das demais; no terceiro momento, a classe 1 foi separada das demais classes; no quarto momento, a classe 7 se diferenciou; na quinta partição, houve a separação das classes 3 e 4;e, na sexta partição, as classes 5 e 6 se separaram, encerrando o processo.

A classe 6 (fator 1) obteve o maior percentual de representação do corpus (18,18\%), na qual a visão do profissional de saúde como agente apenas do Programa Mais Médicos foi expressa nos relatos dos participantes, ao mesmo tempo que eles apontaram que "fazer" $\left(x^{2}=20,9 ; p<0,0001\right)$ "também" $\left(x^{2}=11,6 ; p=0,0006\right)$ a "residência" $\left(x^{2}=9,7 ; p<0,001\right)$ poderia ser uma forma de minimizar essa ameaça.

As classes 1, 2 e 4 apresentaram, cada uma, 15,15\% de representação do corpus. A classe 1 (fator 2 ) representou como ameaça os problemas na "gestão" $\left(x^{2}=4,3 ; p=0,003\right)$ dos equipamentos da saúde, que dificultaram o aprendizado do "aluno" $\left(x^{2}=4,1 ; p=0,004\right)$. 
Figura 3. Nuvens de Palavras das classes geradas na CHD do corpus oportunidades

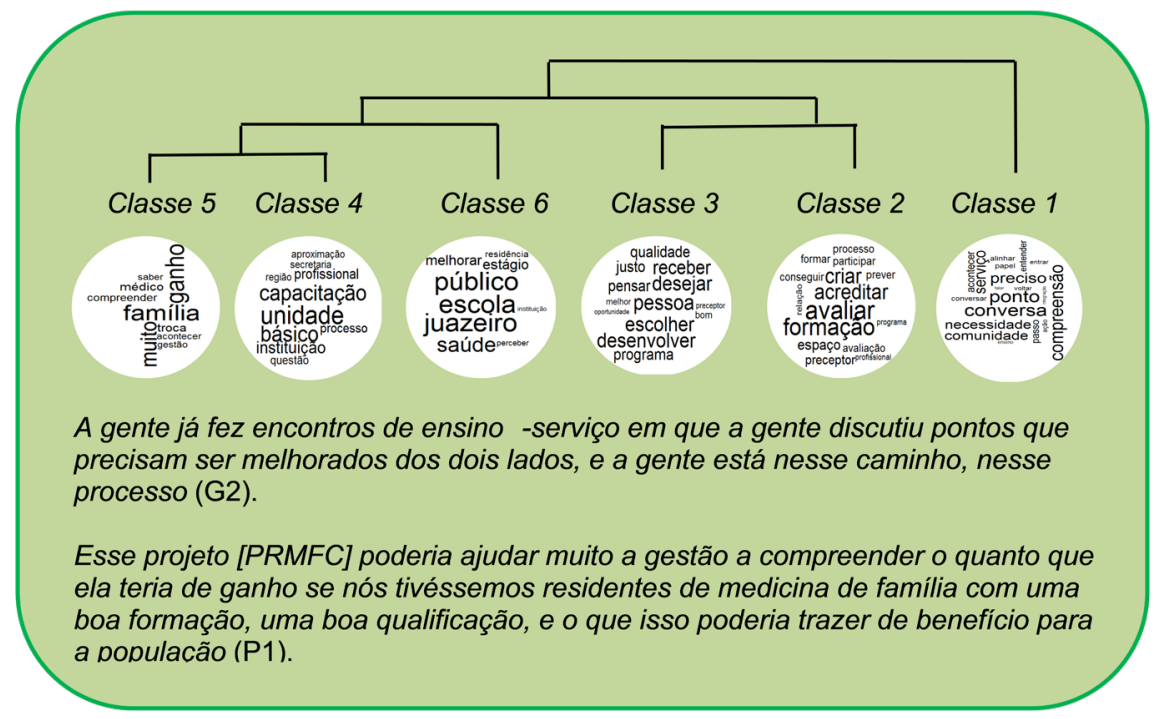

A classe 2 (fator 3 ) evidenciou a problemática de os profissionais não se apresentarem capacitados para que possam atuar como preceptores, na qual os participantes consideraram uma ameaça que poderia ser minimizada por meio da "qualificação" $\left(x^{2}=18,5 ; p<0,0001\right)$ realizada com os profissionais da "atenção primária" $\left(x^{2}=25,5 ; p<0,0001\right)$.

A classe 4 (fator 4) trouxe como ameaça a não compreensão de profissionais acerca do PRMFC, em que o "próprio" $\left(x^{2}=4,3 ; p=0,03\right)$ "profissional de "saúde" $\left(x^{2}=6,81\right.$; $p=0,009$ ) é o maior agente desse processo.

As classes 3, 5 e 7 apresentaram, cada uma, 12,12\% de representação do corpus. A classe 3 (fator 5 ) reportouse à gestão desarticulada do PRMFC, com efeitos diretos sobre o "aluno" $\left(x^{2}=14,22 ; p<0,0001\right)$, sendo corroborada pela inexistência de momentos para análise conjunta que favorecessem espaços para "reflexão" $\left(x^{2}=9,2 ; \quad p=0,002\right)$ pelas "instituições formadoras" $\left(x^{2}=9,2 ; p=0,002\right)$, conforme evidenciado na classe 5 (fator 6).

A classe 7 (fator 7) apresentou como ameaça a estrutura precária dos serviços de saúde, destacando que a "estrutura" $\left(x^{2}=9,2 ; p=0,002\right)$ das "unidades" $\left(x^{2}=16,9 ; p<0,0001\right)$ na quais ocorre a execução do PRMFC necessita de melhor adequação para atingir os propósitos formativos.

As NPs originadas e os discursos dos profissionais complementaram os achados centrais desse corpus (Figura 4).

Decorrente da análise efetuada nos dendrogramas resultantes das CHD foi possível desenhar a matriz SWOT (Figura 5) contendo os cenários interno e externo do PRMFC, aqui representados pelos quatro eixos e seus respectivos fatores.

Figura 4. Nuvens de Palavras das classes geradas na CHD do corpus ameaças

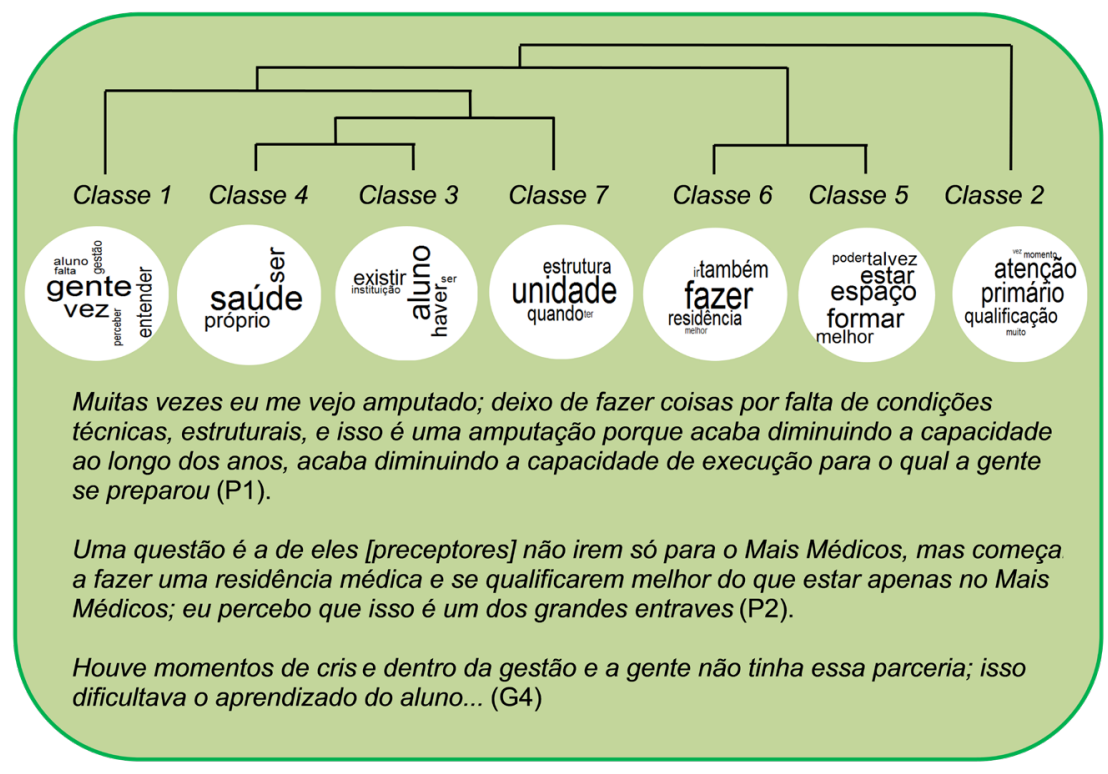


Figura 5. Matriz SWOT do Programa de Residência em Medicina de Família e Comunidade

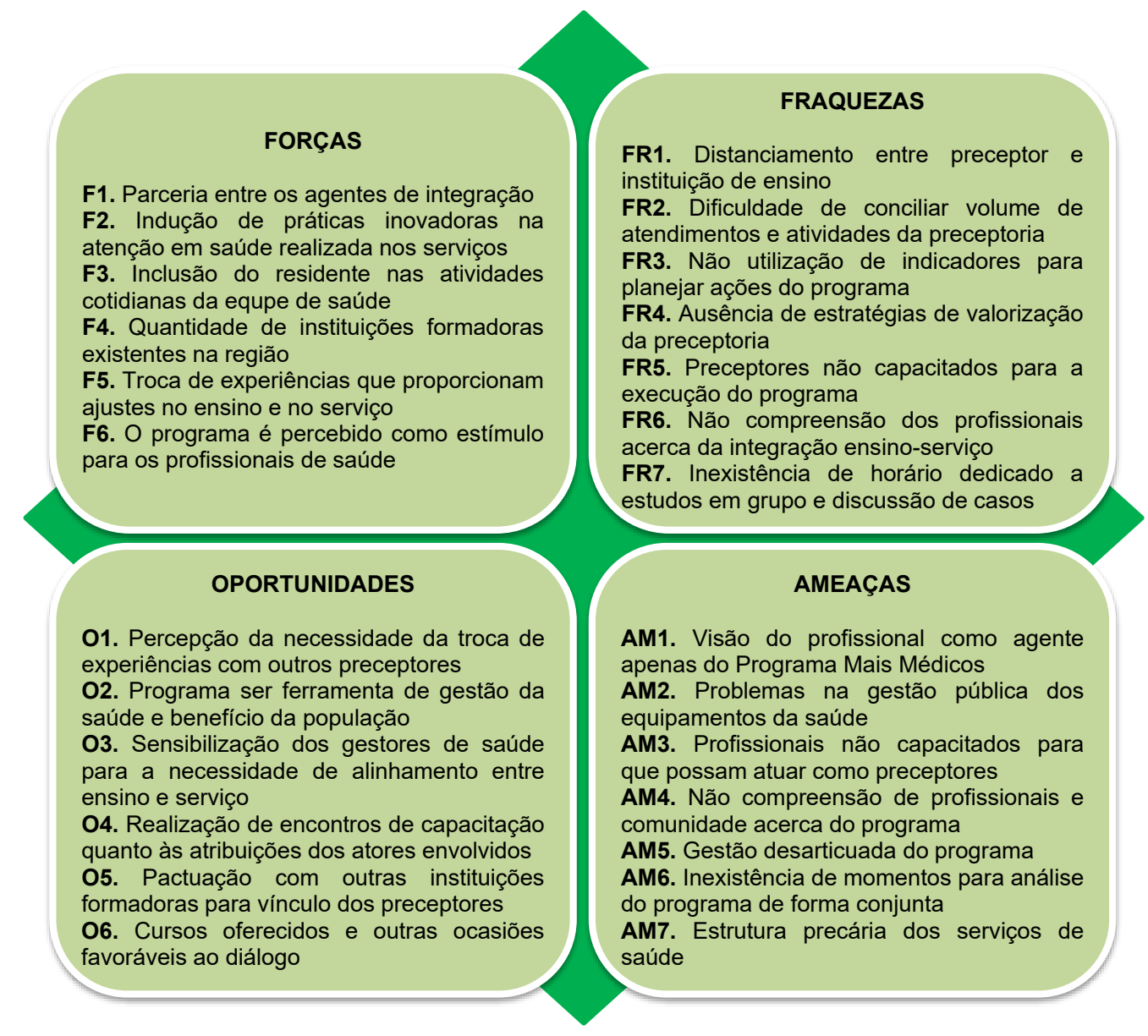

\section{DISCUSSÃO}

A residência médica é uma modalidade de especialização apontada como importante estratégia para a formação de profissionais para atuação no SUS $^{16}$, sendo um período de desenvolvimento de competências essenciais para o trabalho com base nas necessidades e demandas reais em saúde.

Apesar das limitações e dos desafios no processo de integração entre academia e serviço, quando ocorre aproximação entre os envolvidos nesse processo, também ocorrem mudanças na prática profissional de todos, em uma relação de benefício mútuo, com sentimento de pertencimento e corresponsabilização, criando um movimento contínuo de transferência de recursos cognitivos, científicos e subjetivos ${ }^{17,18}$.

Sob outro enfoque, a inserção do residente no campo de práticas mobiliza elementos que interferem positivamente na atuação profissional. Esse residente passa a ser figura central no processo de reestruturação do serviço ao induzir a implantação de novas ações, que vão desde o desenvolvimento de novas práticas até a capacitação dos profissionais da rede para docência. Essa reciprocidade, à medida que aprimora e aperfeiçoa a formação profissional do médico, também promove reflexões e mudanças positivas na gestão do cuidado em saúde dos demais profissionais atuantes naquela realidade.

Os termos centrais do corpus forças compuseram o primeiro eixo da matriz SWOT e contemplaram: parceria entre os agentes de integração e inclusão do residente no cotidiano das equipes com troca de experiências, que impactaram o ensino e o serviço. Esses pontos fortes, por constituírem uma característica interna que favoreceu o alcance dos objetivos ${ }^{19}$, merecem ser considerados como tudo aquilo a ser mantido ou prosperado no âmbito das relações e das ações a serem executadas no íntimo do PRMFC. Os agentes de integração mais envolvidos nesse processo foram os preceptores e os residentes.

As características do relacionamento desenvolvido entre preceptor e residente, e citadas aqui como algo positivo, retratam sua relevância para o trabalho coletivo na Estratégia Saúde da Família $^{20}$, mediado pela valorização do conhecimento do educando, em que o preceptor desenvolve a crítica educadora, em constante processo de elaboração e reelaboração do saber-fazer, de modo a horizontalizar essa relação ${ }^{21}$. Nesse sentido, as expressões evidenciadas nesse eixo refletiram como os agentes do PRMFC vêm tecendo suas relações exitosas no direcionamento da formação médica especializada. 
O distanciamento entre instituição de ensino e preceptor foi anunciado no estudo como um desafio a ser superado pelos agentes de integração, e a relação existente entre estes pouco reflete novas práticas ou aperfeiçoamento do processo de trabalho, pelo fato de não haver integração entre o residente e os profissionais da equipe. Entretanto, sua participação no envolvimento avaliativo do PRMFC poderia promover maior integração, conferindo sentimento de pertencimento ao processo.

As fraquezas centrais evidenciadas no estudo envolveram distanciamento entre preceptor e instituição de ensino, dificuldade de conciliar o volume de atendimentos com as atividades da preceptoria e ausência de estratégias de valorização da preceptoria, e compuseram o segundo eixo da matriz SWOT. Esses pontos fracos evidenciaram aspectos internos do PRMFC, delineados como o conjunto de deficiências capazes de prejudicar ou dificultar a execução dos objetivos ${ }^{22}$, os quais realçaram o preceptor como o agente de integração mais influente nesse contexto, uma vez que esse profissional configura-se como mediador da aprendizagem e produção de saberes no próprio ambiente de trabalho ${ }^{23}$.

As considerações apresentadas no eixo fraquezas se manifestaram como um aspecto negativo, pois a desvalorização da preceptoria foi um reflexo da deficiência da integração entre a instituição de ensino e o serviço, o que provoca os agentes envolvidos a promover mudanças desde a fase do planejamento até a fase de execução e assim resultar em melhorias efetivas no desenvolvimento e na qualidade do PRMFC.

O preceptor, como agente promotor e facilitador da aprendizagem, apresenta-se como um elo entre os acadêmicos, os demais profissionais da unidade e os usuários do serviço. Os vínculos desenvolvidos por meio das parcerias de trabalho interprofissional favorecem aos residentes o desenvolvimento de habilidades essenciais para a atuação na atenção primária à saúde, possibilitando, especialmente, a integralidade do cuidado, em conformidade com os princípios do sistema público de saúde. Nesse ínterim, as unidades básicas de saúde são um lócus privilegiado na promoção da construção e da troca de saberes, tanto no âmbito organizacional quanto no assistencial, pois implica uma nova lógica de organização do processo de trabalho, oportunizando novas experiências e aprendizados aos atores envolvidos ${ }^{24}$.

No âmbito geral, oportunidades podem ser compreendidas como situações externas, podendo ser atuais ou potenciais, que contribuam para o alcance dos objetivos e a melhoria do contexto identificado ${ }^{25}$. Contudo, essas potencialidades requerem vontade política dos agentes de integração para que possam evitar posições dissonantes e assim promover transformações efetivas ${ }^{26}$.
As oportunidades evidenciadas constituíram o terceiro eixo da matriz SWOT e abrangeram: percepção da necessidade da troca de experiências com outros preceptores; perspectiva de o PRMFC servir como ferramenta de gestão da saúde; e pactuação com outras instituições formadoras para o oferecimento de cursos e outras situações favoráveis ao diálogo.

A estrutura precária dos serviços, tanto de recursos materiais quanto humanos, e a deficiência na interlocução entre os agentes de integração do PRMFC (gestão-instituição de ensino-serviço) sinalizam a urgente necessidade de criação de espaços para o compartilhamento de demandas e experiências, de modo a favorecer esclarecimentos sobre o papel e a função de cada um dos envolvidos nessas comissões ${ }^{27}$, especialmente quanto ao papel do preceptor.

As ameaças verificadas no estudo formaram o quarto eixo da matriz SWOT e englobaram: visão do profissional como agente apenas do Programa Mais Médicos; e os problemas na gestão dos equipamentos da saúde, com reflexos sobre a estrutura dos serviços, a gestão do PRMFC e a qualidade da formação. Tais situações podem prejudicar a execução dos objetivos formativos dos residentes e a inter-relação com outros programas e cursos de saúde que compartilham os mesmos campos de prática ${ }^{28,29}$, pois impactam diretamente o ambiente interno e não são controláveis ${ }^{30}$. Assim, essas ameaças devem ser consideradas de modo permanente no planejamento das ações do programa, com o intuito de mantê-las sempre à margem dos processos que envolvam o ambiente avaliado.

Na perspectiva de que a identificação pormenorizada dos eixos da SWOT pode contribuir para a melhoria do desempenho da unidade na qual o gestor de saúde atua ${ }^{31}$, considerada aqui como sendo o PRMFC cujos gestores se caracterizam pelos secretários de saúde, dirigentes de ensino, preceptores, residentes e pela comunidade, foi possível evidenciar os aspectos positivos e negativos que orientaram os próximos passos para elaboração do plano de fortalecimento da relação entre instituições de ensino e de saúde.

O diagnóstico situacional do PRMFC estudado, confrontadocomaliteratura revisada, favoreceu o entendimento acerca do contexto do PRMFC e balizou a construção de uma proposta para o fortalecimento da integração ensinoserviço, que se estruturou em três pilares: 1.fortalecimento da integração ensino-serviço; 2.fortalecimento do PRMFC; e 3.fortalecimento da preceptoria.

Com essas ponderações, vislumbra-se a matriz SWOT como uma ferramenta oportuna para a análise situacional dos processos que envolvem a integração ensino-serviço, de modo a contribuir para a elaboração de melhorias na interlocução entre os atores envolvidos e minimizar conflitos e desafios. Além disso, como ocorreu nesta pesquisa-intervenção, essa 
ferramenta pode ser utilizada em outros cenários e programas de formação em saúde.

\section{CONCLUSÕES}

A análise SWOT possibilitou identificar aspectos específicos que fazem parte do cotidiano do PRMFC, tanto fatores internos quanto externos, ressaltando pontos positivos, mas também aspectos que precisam ser modificados e/ou superados pelos envolvidos.

Todas as reflexões aqui apresentadas, como forças, fraquezas, oportunidades e ameaças, indicam pontos que podem provocar mudanças reais e positivas na melhoria da gestão e na execução do cuidado, ou seja, ao apontarem os aspectos falhos, dão espaço para a reorganização e remodelação de fatores que fortalecerão e favorecerão uma melhor e maior integração entre o ensino, o serviço de saúde e a gestão das instituições envolvidas.

Destaca-se, como limitação para o estudo, a não inclusão de outros atores envolvidos no PRMFC como participantes desta pesquisa, como dirigentes de ensino, residentes e comunidade, o que certamente ampliaria o olhar sobre essa realidade e agregaria outros objetivos e outras ações estratégicas ao plano para fortalecimento do PRMFC. Nesse sentido, aponta-se como perspectiva futura a necessidade de inclusão desses atores em estudos como o aqui apresentado.

\section{CONTRIBUIÇÃO DOS AUTORES}

Maria de Fátima Antero Sousa Machado e Anderson Milfont Feitosa de Oliveira contribuíram na concepção e no delineamento do estudo, na coleta dos dados, na análise e interpretação dos resultados,e na elaboração e revisão crítica do conteúdo do manuscrito. Maria Rosilene Cândido Moreira e Samyra Paula Lustoza Xavier contribuíram na análise e interpretação dos resultados, e na elaboração e revisão crítica do conteúdo do manuscrito. Todos os autores aprovaram a versão final do manuscrito e declaram-se responsáveis por todos os aspectos do trabalho, garantindo sua precisão e integridade.

\section{CONFLITO DE INTERESSES}

Os autores declaram não haver conflito de interesses neste estudo.

\section{FINANCIAMENTO}

Declaramos que não houve financiamento para a realização desta pesquisa.

\section{REFERÊNCIAS}

1. Vedruscolo C, Silva MT, Silva MEK. Integração ensino-serviçocomunidade na perspectiva da reorientação da formação em saúde. Sustinere.2017;5(2):245-59.

2. Brasil. Lei $\mathrm{n}^{\circ} 12.871$. Institui o Programa Mais Médicos, altera as Leis no 8.745, de 9 de dezembro de 1993, e n6.932, de 7 de julho de 1981, e dá outras providências. Diário Oficial da União,Brasília, DF;22 out. 2013; Seçãol, p. 1-4.

3. Brasil. Decreto $n^{\circ} 80.281$, de 5 de setembro de 1977. Regulamentação da Residência Médica e criação da Comissão Nacional de Residência Médica. Diário Oficial da União,Brasília, DF;1977.

4. Pizzinato A, Gustavo AS, Santos BRL, Ojeda BS, Ferreira E, Thiesen FV, et al.Integration between learning and health services as a strategy for professional training in the Unified Health System. Rev Bras Educ Med. 2012;36(1):170-7.

5. Macêdo NB, Albuquerque PC, Medeiros KR. O desafio da implementação da educação permanente na gestão da educação na saúde. Trab Educ Saúde.2014;12(1):379-401.

6. Zarpelon LFB, Terencio ML, Batista NA. Integração ensino-serviço no contexto das escolas médicas brasileiras: revisão integrativa. CienSaude Colet. 2018;23(12):4241-8.

7. Antunes MTP, Mendonça Neto OR, Vieira AM. Pesquisa intervencionista e mestrados profissionais: perspectivas de sua prática nos cursos da área de gestão. Indagatio Didactica.2016;8(3):53-68. [acesso em 11 abr 2018]. Disponível em: https://proa.ua.pt/index.php/id/article/view/2569.

8. Rosenau LS, Costa RR, Trevisan TS. Pesquisa-ação e participante: suas contribuições para o conhecimento científico. Anais do V Seminário de Pesquisa em Educação da Região Sul - V ANPED SUL; 2004. [acesso em 21 abr 2019]. Disponível em: https://docplayer.com.br/15137188-Pesquisa-acao-eparticipante-suas-contribuicoes-para-o-conhecimento-cientifico.html.

9. Camargo BV, Justo AM. IRAMUTEQ: um software gratuito para análise de dados textuais. Temas Psicol. 2013;21(2):513-8.

10. Salvador PTCO, Gomes ATL, Rodrigues CCFM, Chiavone FBT, Alves KYA, Bezerril MS, et al.Uso do software IRAMUTEQ nas pesquisas brasileiras da área da Saúde: uma scoping review. Rev Bras Promoç Saúde.2018;31(supl):1-9.

11. Andrade Júnior EO, Andrade EO. Lexical analysis of the Code of Medical Ethics of the Federal Council of Medicine. AMB Rev Assoc Med Bras. 2016;62(2):123-30.

12. Camargo BV, Justo AM. Tutorial para uso do software IRAMUTEQ 2018. [acesso em 08 jan 2019]. Disponível em: http://iramuteq.org/ documentation/fichiers/tutoriel-portugais-22-11-2018.

13. Mendes FRP, Zangao MOB, Gemito MLGP, Serra ICC. Social representations of nursing students about hospital assistance and primary health care. Rev Bras Enferm. 2016;69(2):321-8.

14. Kummer DC, Silveira, RLL. A importância da matriz SWOT (FOFA) no contexto dos planos estratégicos de desenvolvimento do Rio Grande do Sul. Revista Jovens Pesquisadores. 2016;6(1):101-15.

15. Lacerda JT, Botelho LJ, Colussi CF. Carlos Matus e o Planejamento Estratégico Situacional (PES). [acesso em ago 2018]. Disponível em https://unasus2.moodle.ufsc.br/pluginfile.php/19821/mod_resource/ content/2/un2/top3_1.html.

16. Berger CB, Dallegrave D, Castro Filho ED, Pekelman R. A formação na modalidade residência médica: contribuições para a qualificação e provimento médico no Brasil. RevBrasMedFam Comunidade. 2017;12(39):1-10.

17. Domingos CM, Nunes EFPA, Carvalho BG. Potential of multiprofessional residency for family healthcare: the view of healthcare workers. Interface (Botucatu). 2015;19(55):1221-32.

18. Pinheiro LCR, Carvalho RB, Viana PFS. Práticas de integração ensinoserviço-comunidade e a formação em Odontologia: possíveis conexões e fluxos no ensino na saúde. Rev. ABENO.2018;18(4):148-59. 
19. Travassos PFS, Vieira FO. Aplicação da análise SWOT na preparação do processo de avaliação institucional interna (auto-avaliação) realizada pela IES. Revista Eletrônica de Administração. 2011;10(2):1-14.

20. Arnemann CT, Kruse MHL, Gastaldo D, Jorge ACR, Silva AL, Margarites AGF, et al. Preceptor's best practices in a multiprofessional residency: interface with interprofessionality. Interface (Botucatu). 2018;22(Suppl 2):1635-46.

21. Barreto VHL, Monteiro RCS, Magalhães GSG, Almeida RCC, Souza LN. Papel do preceptor da atenção primária em saúde na formação da graduação e pós-graduação da Universidade Federal de Pernambuco: um termo de referência. Rev Bras Educ Med. 2011;35(4):578-83.

22. Lamenha AAR, Patrício SRR. O planejamento estratégico utilizando a análise SWOT como recurso para a tomada de decisão: uma aplicação prática em uma empresa de saúde de pequeno porte. Olhares Plurais, Revista Eletrônica Multidisciplinar. 2014;1(10):23-45.

23. Lima PAB, Rozendo CA. Challenges and opportunities in the Pró-PETHealth preceptorship. Interface (Botucatu). 2015;19(Suppl 1):779-91.

24. Pagani R, Andrade LOM. Preceptoria de território, novas práticas e saberes na estratégia de educação permanente em saúde da família: o estudo do caso de Sobral, CE. Saúde Soc. 2012;21(1):94-106.

25. Fernandes DR. Uma visão sobre a análise da matriz SWOT como ferramenta para elaboração da estratégia. UNOPAR Cient., Ciênc. Juríd. Empres. 2012;13(2):57-68.
26. Sarti TD, Fontenelle LF, Gusso GDF. Panorama da expansão dos Programas de Residência Médica em Medicina de Família e Comunidade no Brasil: desafios para sua consolidação. RevBrasMedFam Comunidade. 2018;13(40):1-5.

27. França T, Medeiros KR, Belisario AS, Garcia AC, Pinto ICM, Castro JL, et al. Política de Educação Permanente em Saúde no Brasil: a contribuição das Comissões Permanentes de Integração Ensino-Serviço. Cien Saude Colet.2017;22(6):1817-28.

28. Alessio MM, Sousa MF. Regulação da formação de especialistas: interrelações com o Programa Mais Médicos. Physis. 2016;26(2):633-67.

29. Storti MMT, Oliveira FP, Xavier AL. A expansão de vagas de residência de Medicina de Família e Comunidade por municípios e o Programa Mais Médicos. Interface (Botucatu).2017;21(supl1):1301-14.

30. Souza LPS, Souza AMV, Pereira KG, Figueiredo T, Bretas TCS, Mendes MAF, et al. Matriz SWOT como ferramenta de gestão para melhoria da assistência de enfermagem: estudo de caso em um hospital de ensino. Revista Eletrônica Gestão\& Saúde.2013;4(1):1633-43.

31. Barbosa NCT, Cordeiro BC, Abrahão AL, Xavier ML, Carvalho RS, Silva ROC et al. Educação em saúde: o uso da matriz SWOT para análise de projetos. Rev Enferm UFPE. 2017;11(11):4298-304. 